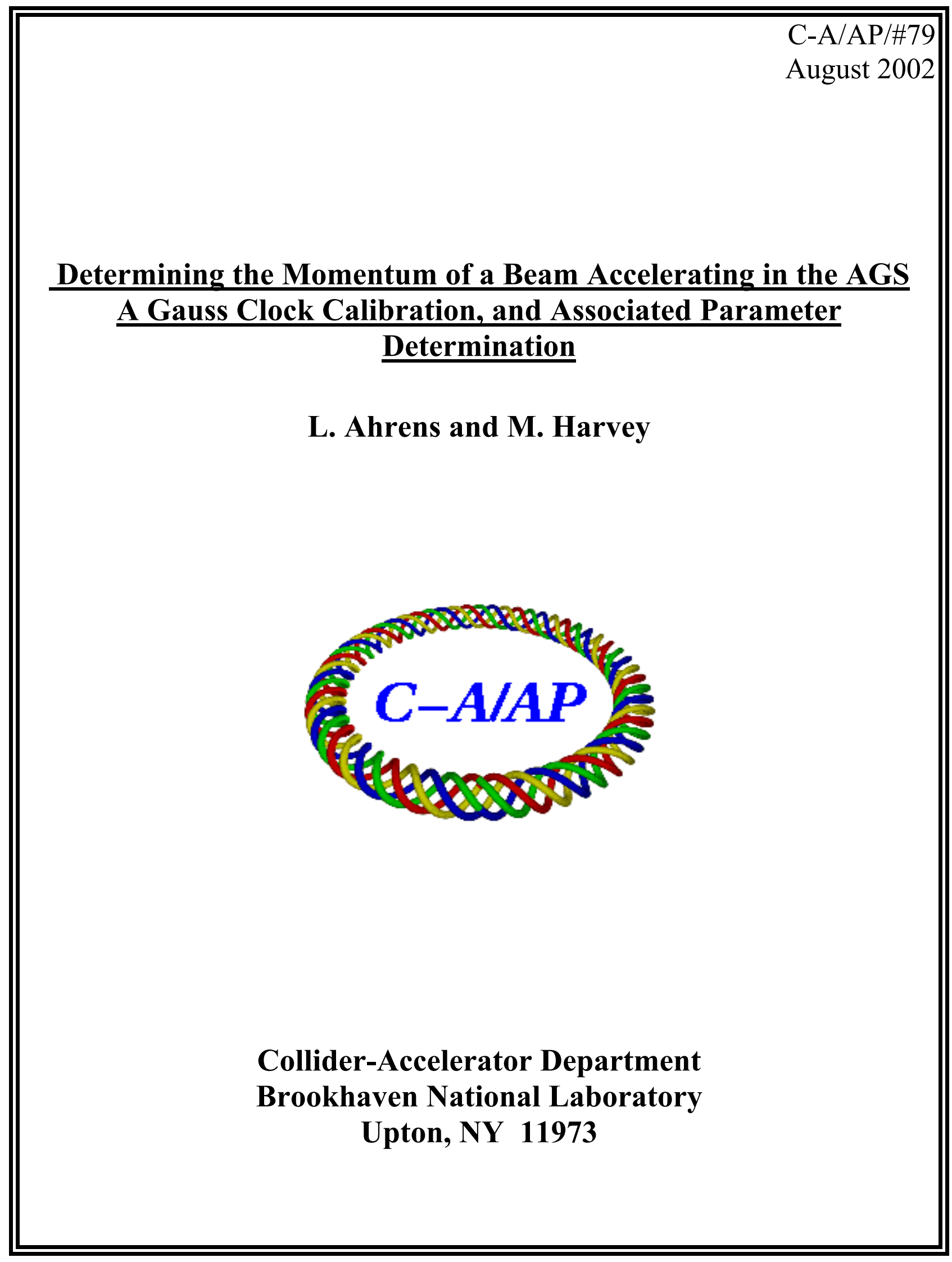


C-A AP Note-79 6Aug02

L. Ahrens and M. Harvey

\section{Determining the Momentum of a Beam Accelerating in the AGS A Gauss Clock Calibration, and Associated Parameter Determination}

\section{Introduction}

Knowing the momentum of the AGS beam is frequently important and occasionally critical for acceptable performance of the accelerator. In particular, the sensitivity to momentum-dependent gymnastics of a polarized proton beam makes momentum knowledge a requirement. The beam momentum is defined if any two of the three parameters (average magnetic field, path length, revolution frequency) can be determined. One of these, the frequency, is measurable directly. The other two come with baggage. Deviations in the path length are directly related to the offsets measured by the beam position monitors[1] which are usually simplified into a single average offset (the $R$ below), relative to the standard orbit, where the standard orbit has length $2 \pi^{*} R_{0}$. [2] The average magnetic field is related to the integration of the voltage produced in a coil located in the AGS reference magnet[3] as the magnetic field ramps during the acceleration cycle. This integral is made available to the world through the Gauss Clock[4], with up ticks and down ticks (Gauss Clock Counts GCC) coming as the field increments or decrements by equal small amounts. So both the radius and the field measurements require from beam measurements, dead reckoning, or both the determination of an offset and a "slope", and some assurance that this linear approximation is adequate. This note starts from a revisit of the Gauss Clock calibration, but then necessarily continues into the beam position monitor space to come to an encouraging picture of our capability to determine the beam momentum. The work is not fundamentally different from measurements made and described by Weisberg[5] in 1978 and has been revisited several times since then. What have happened since this method was last used are upgrades to both the Gauss Clock system (by Joe Geller et al.) and the electronics for the AGS orbit acquisition and display system (by Kevin Smith et al.).

\section{AGS Gauss Clock Calibration}

The "Gauss Clock" is an electronic system that carefully integrates the voltage from a coil and outputs a "tick" each time that integral increases to a certain value. The system then resets and starts a new integration. The coil resides in the AGS reference magnet and is designed such that the voltage is proportional to the change in (magnetic field $x$ path length) that a particle accelerating along the reference orbit in the AGS would see. If in a "with beam" situation we can independently measure the momentum of the accelerating particles along with the clock ticks, then the clock can be calibrated. That independent momentum measurement requires measuring the frequency and path length of the particle's motion around the synchrotron. Understanding subtle systematics of all of the contributing measurements is important if the calibration is to be pushed to the limits. To this end many measurements are made as the magnetic field is increased. Because the Gauss Clock deals only with changes in field through the coil, its calibration can be assumed to be independent of the magnitude of the field. Plotting Gauss Clock counts against the measured beam momentum (corrected to be on the reference orbit) should give a straight line. 
To calibrate the Gauss clock we obtained measurements of the gauss clock counts, GCC, the radius, $R$, and the $\mathrm{rf}$ frequency (harmonic $h$ ), $f$, simultaneously. With the measured values for radius and frequency we find the velocity

$$
v=\frac{2 \pi\left(R+R_{0}\right) f}{h} .
$$

From the velocity we get

$$
\begin{aligned}
& \gamma=\frac{1}{\sqrt{1-\beta^{2}}} \\
& E=E_{0} \gamma
\end{aligned}
$$

then

$$
p(R)=E \beta
$$

and using the differential behavior of momentum with radius at fixed magnetic field:

$$
p\left(R_{0}\right)=p(R)-\frac{p(R) R \gamma_{t r}^{2}}{R_{0}}=p(R)\left[1-\frac{R}{R_{0}} \gamma_{t r}^{2}\right]
$$

from this we can calculate the field

$$
B\left(p\left(R_{0}\right)\right)=\frac{p\left(R_{0}\right)}{c \rho} \times 10^{11}
$$

where

$$
\begin{aligned}
& h=\text { harmonic number }=6 \\
& \beta=v / \mathrm{c} \\
& E_{0}=\text { proton rest mass energy }=0.9382723 \mathrm{Gev} / \mathrm{c}^{2} \\
& c=2.997925 \times 10^{10} \mathrm{~mm} / \mathrm{sec}
\end{aligned}
$$

and where

$$
\begin{aligned}
& \gamma_{t r}=8.5 \\
& \rho=85378.34 \mathrm{~mm} . \\
& R_{0}=\text { central orbit of the AGS }=128452.6 \mathrm{~mm}
\end{aligned}
$$

The last three "constants" are the conventional values for basic properties of the AGS, and open to challenge - to the extent that the analysis is sensitive to their values.

\section{Data and Analysis}

Two data taking runs are covered by this report. The data-taking technique was the same for both. One run occurred during the polarized proton run, early December 01 , with one bunch of $1 \mathrm{e} 11$ protons accelerating in the AGS. The other, specifically detailed just below, was taken during high intensity proton running (20Apr02) with 6 bunches of $1 \mathrm{e} 13$ protons each accelerating in AGS. 
The MCR3 frequency analyzer (HP 5371A) and the AGS Orbit Display program are set to average over the same ten AGS cycles. Both measurements involve time intervals, or windows, that are centered on the times of interest. We also record the net (i.e. up minus down) GCC for the same cycles. Our three-quantity measurements are taken at $25 \mathrm{~ms}$ intervals over the field's rise time, beginning at 950 ms from AGS T0 and ending at $1800 \mathrm{~ms}$.

Figure 1 is a plot of the extracted $B\left(p\left(R_{0}\right)\right)$ defined above, namely the predicted magnetic field from the orbit and frequency measurements at a given time against GCC together with a least-squares fit to a line. We find that 19.506 GCC equal 1 Gauss, which is close to the design number of 20.

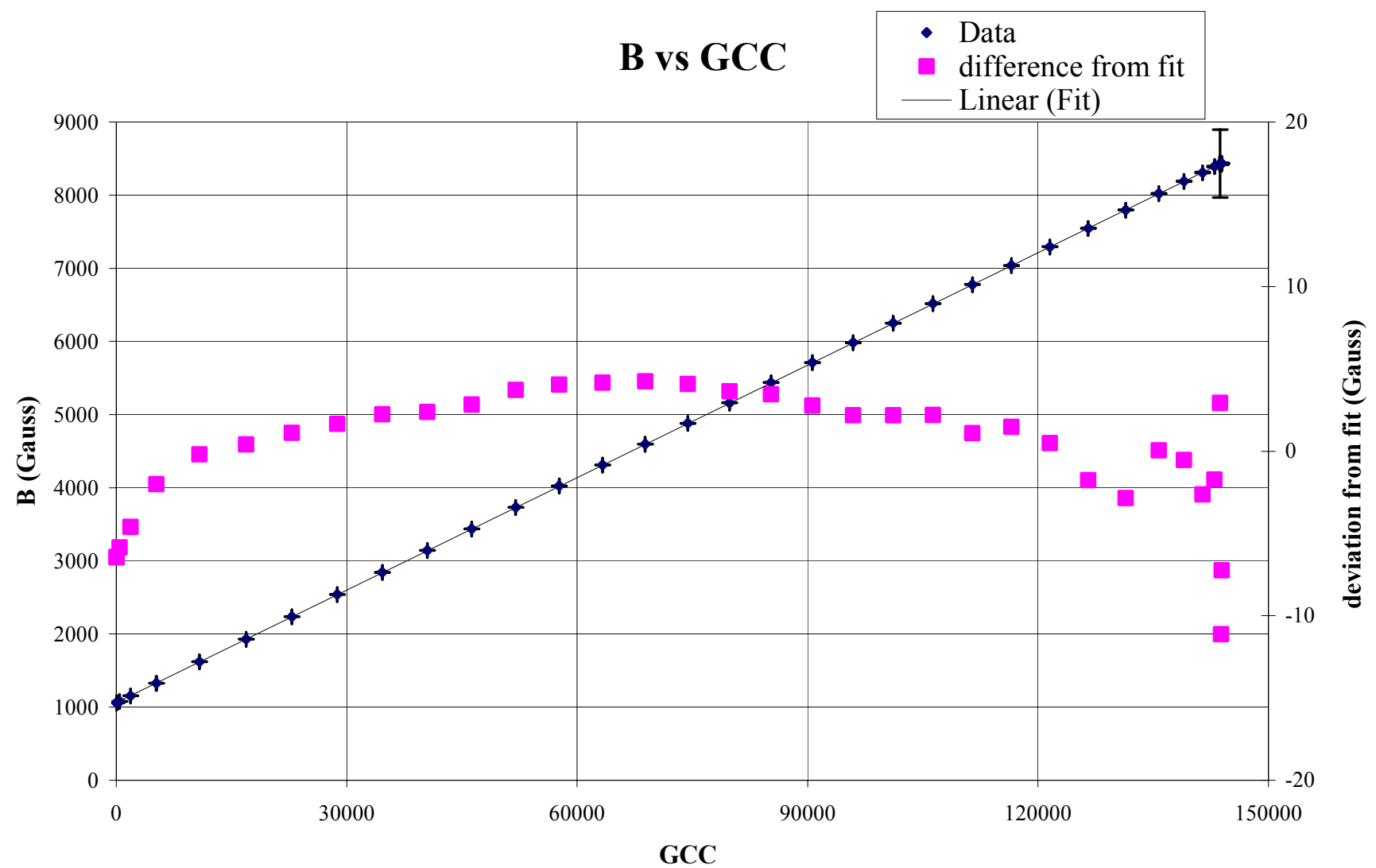

Figure 1. Results of calculated B field verses GCC with linear fit.

Also shown is the deviation from the fit. Note that the deviation, over the central GCC region, shows smooth behavior at the few Gauss level, suggesting a systematic problem. 
The rate at which the AGS magnetic field is changing is a possibly relevant parameter in this study. The data is taken with the Westinghouse motor generator set powering the AGS Main Magnets. To measure the rate of change of magnetic field at each point, GCC measurements were taken $1 \mathrm{~ms}$ before and $1 \mathrm{~ms}$ after each sample time. The average of these two Bdot are plotted vs. GCC in Figure 2.

\section{B dot vs GCC}

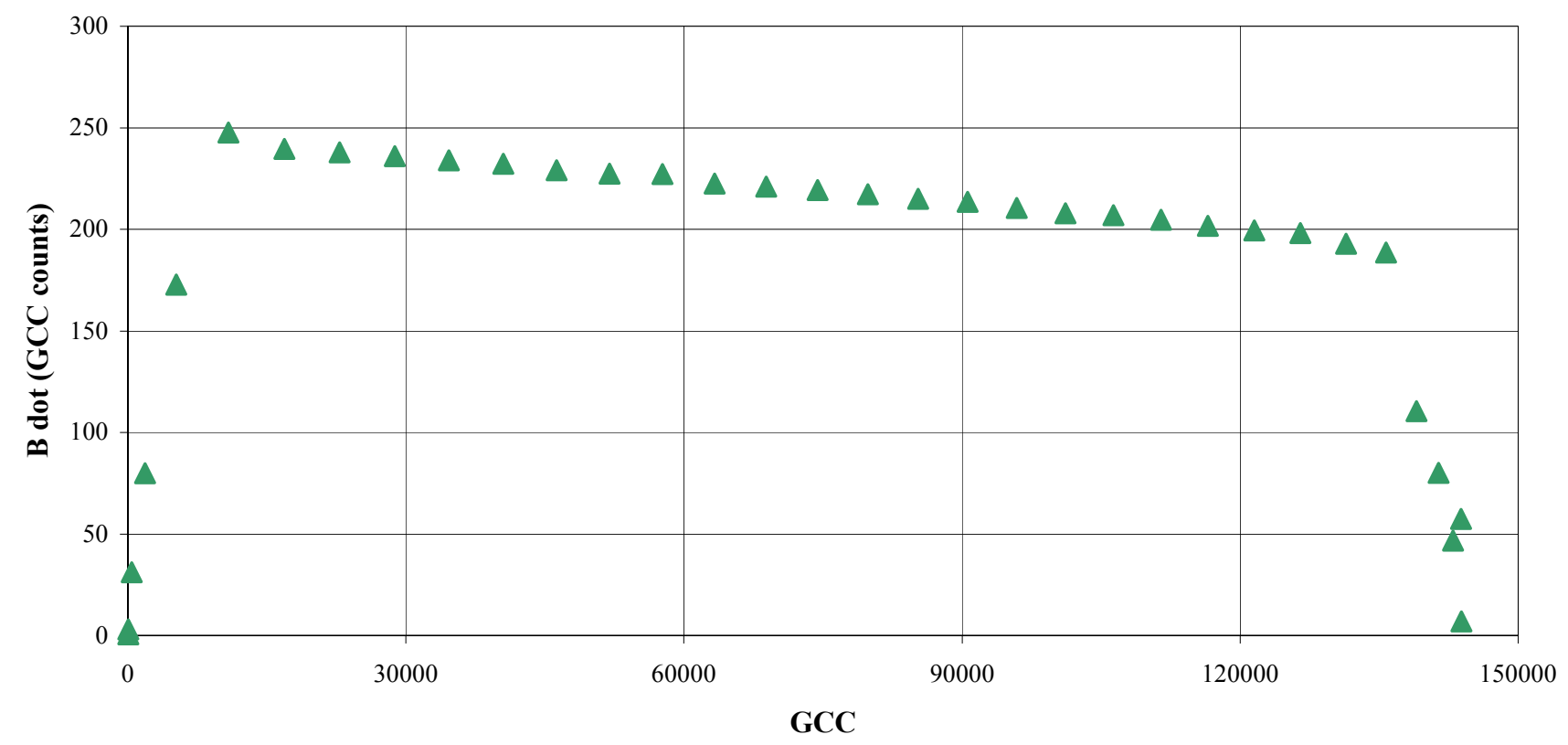

Figure 2. Bdot vs GCC from the end of the dwell field to the beginning of flattop.

For our initial calculations we have taken $R_{0}$, the "predicted" value [2], to be $128452.6 \mathrm{~mm}$. The apparent systematic deviation of the predicted field (from $f$ and $R$ ) relative to the prediction from the GCC (i.e. linear with GCC) that is evident in figure 1 encouraged a search for a cause (see Appendix A). By allowing $R_{0}$ to vary we can find the value for the central orbit which minimized the deviations. Figure 3 is a plot of the square of the difference between measured field and fitted field with changing $R_{0}$, the minimum occurs at $128453.7 \mathrm{~mm}$, i.e. about $1 \mathrm{~mm}$ above the book value and is sensitive at the "few tenths of mm" level. 


\section{Ideal Radius}

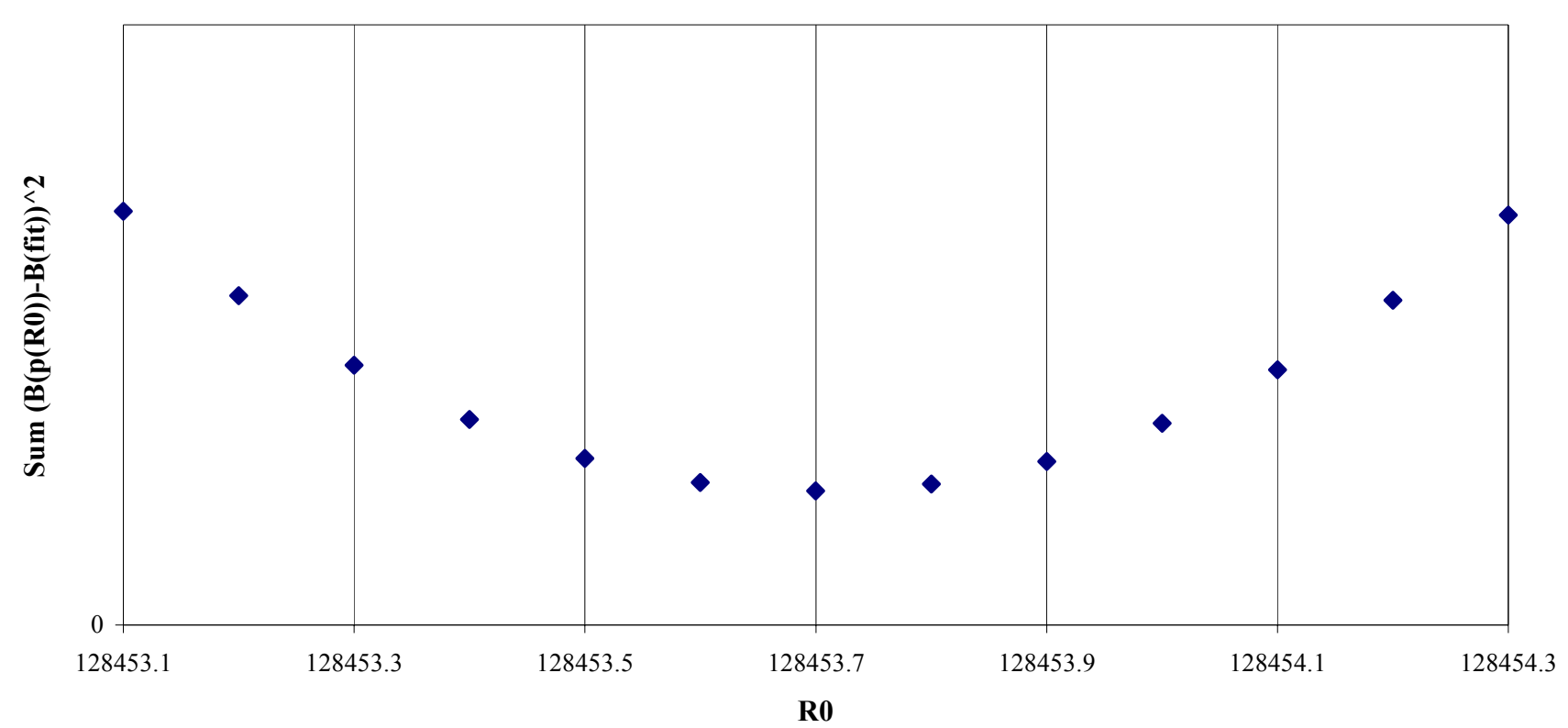

Figure 3. Difference in calculated field and fitted field vs. varying $R_{0}$.

Making this change in the experimental $R_{0}$ and redoing figure 1 produces figure 4 . So now we are affecting both of our momentum determining variables, $R$ and $B$. The GCC calibration changes from $19.506 \mathrm{GCC} /$ Gauss to $19.434 \mathrm{GCC} /$ Gauss and $R_{0}$ increases by $1 \mathrm{~mm}$. Because of polarized proton demands, these changes are actually significant, but equally importantly there comes some comfort that they may be reflecting reality. 


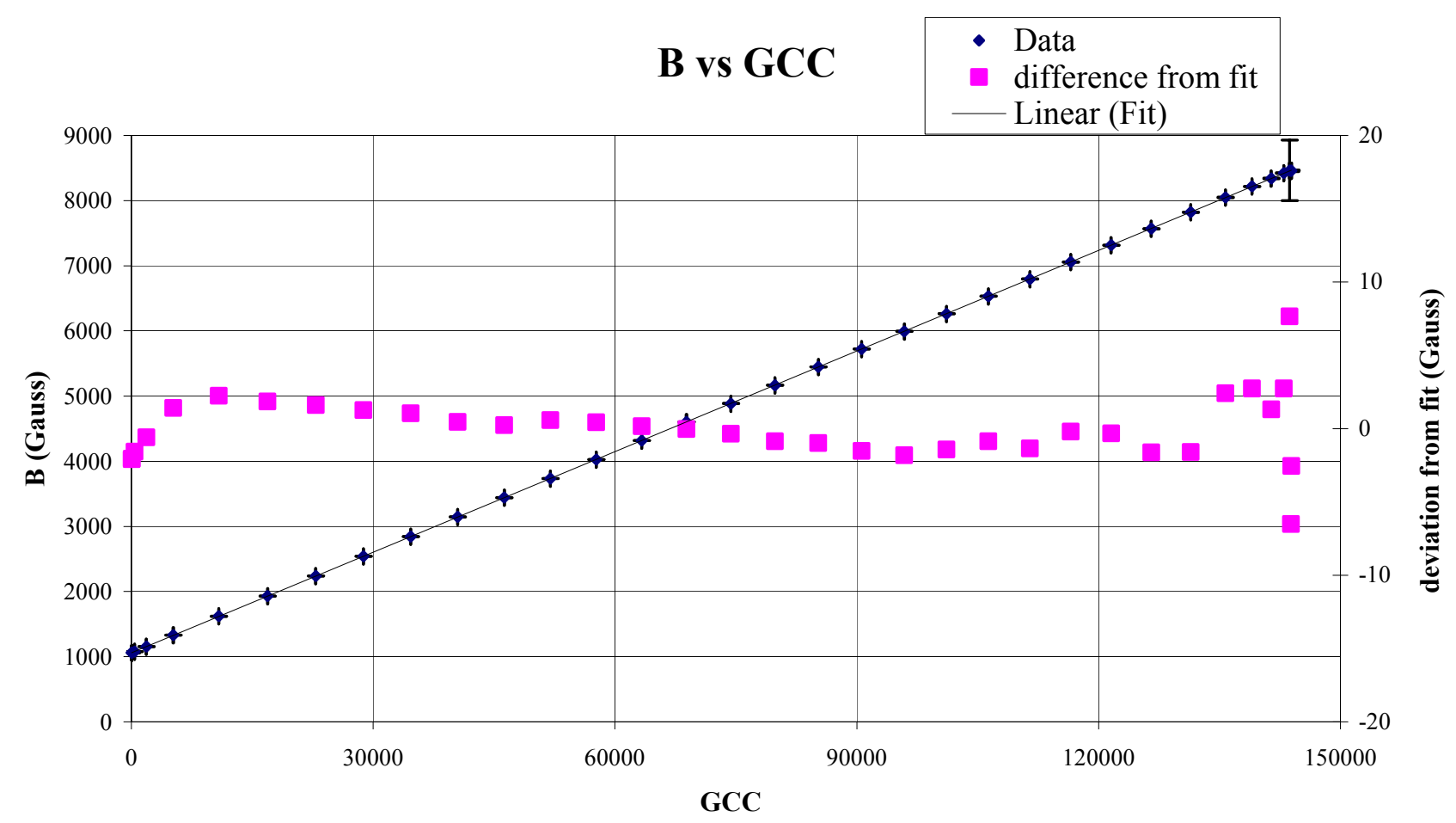

Figure 4. B field verses GCC using $R_{0}$ equal to $128453.7 \mathrm{~mm}$.

This slope gives a value of 19.434 GCC/Gauss.

\section{Setup and procedure for making measurements.}

All measurements are averaged over ten cycles. For the frequency and radial measurements the gate width should be centered on the time of measurement.

GCC are triggered on A.GNRC.REAL.RT to reduce jitter. (The standard AGS Scope triggers have inherent jitter at their clock speed, which is $1 \mathrm{~ms}$ for the times required for this study). Up and down counts are recorded for each cycle. We are interested in the average up-down counts for each time. Additionally, in order to determine $B$ dot take data at $\pm 1 \mathrm{~ms}$ for each time.

Frequency is measured using the HP5371A Frequency and Time Interval Analyzer. For this data set the gate width was set to $1 \mathrm{~ms}$.

The radial measurements are taken using the AGS Orbit Display program. The gate width was $100 \mu$ s.

\section{Discussion (and the answer)}

As has been mentioned above, a second Gauss Clock calibration measurement with polarized beam was carried out a few months before the one just described. When the procedure to adjust $R_{0}$ to 
maximize the goodness-of-fit was applied to that data (which was on fact not done during the polarized run) a GCC slope of $19.50 \mathrm{GCC} / \mathrm{Gauss}$ at the standard Bleser $\mathrm{R}_{0}$ changed to a slope of 19.455 with $\mathrm{R}_{0}$ increasing by $.94 \mathrm{~mm}$ (see appendix A for more details). The two scans end up consistent for a slope of (19.445 +/- .01) GCC/Gauss, and an $R_{0}$ of $128453.6+/-.1 \mathrm{~mm}$. This may underestimate the error on 'truth', but probably not by too much. The two data runs involved very different situations as far as the intensity coped with by the orbit system. The orbit "setup", the pick-up electrodes marked as "bad" etc. was not touched between the two runs, which would seem a necessary condition to extract the same $\mathrm{R}_{0}$, though hopefully not to get the same GCC slope.

Appendix B attempts to "explain" how the three measured variables interact in determining the beam momentum. Included here is the observation that at injection energy in AGS conditions are right the beam is sufficiently nonrelativistic - to allow an adequate momentum estimation using frequency alone - given that the beam is surviving in the machine.

To actually learn the machine magnet field at a particular time in the cycle from the Gauss Clock reading requires more than the average slope (during periods of near constant acceleration). One needs to know the field at the start of the interval across which the Gauss clock counts are counted, and estimate the error from any regions of different slope. That we can come to an absolute measure at or even shortly after injection using frequency alone, gets us over the region of changing $B$ dot at the start of acceleration. There remains the interval going into the extraction flat top. That error has not been carefully estimated. It is relevant for the determination of the extraction momentum.

Appendix $\mathrm{C}$ explores one aspect of the frequency measurement error. With care this error can be improved over what we have done in our scans. However as Appendix B, figure B3, shows, frequency alone is not adequate to learn the momentum except very early in the cycle.

\section{Future Work}

The general self-consistency of the analysis presented here suggests that the space can be pushed further. Asking whether the Gauss Clock has a slightly different slope for different rates of acceleration is appropriate. Studying how the effective BPM system $R_{0}$ changes during a given cycle may be required and difficult since the answer comes from watching Gauss clock response as the beam velocity changes. One maneuver during acceleration expected to affect the derived $\mathrm{R}_{0}$ is the transition gamma jump, since the machine dispersion deformation required to produce the jump effectively changes the individual BPM calibrations. There is some hint of this in the present data. The measurement of pulseby-pulse variation of the machine should allow better error analysis and may teach us more about the machine stability. The tools to do pulse-by-pulse easily don't quite exist. We need the radius from the orbit program, the frequency from the frequency meter, and the Gauss counts, each saved along with its AGS cycle number to do the correlation.

\section{References}

[1] D.J.Ciardullo and J.M.Brennan, Acc Div Tech Note No. 411, The AGS BPM System Upgrade, 31Jan95

[2] E. Bleser, Acc Div Tech Note No. 215, Where are the AGS Magnets?, 20May85 
[3] A. Warner and W. van Zwienen, Acc Div Tech Note No.363, The Design of the Dipole Pick-up Coil for use with the AGS Gauss Clock, 5 Aug92

[4] J. Geller, Booster Tech Note No. 175, A Digital Voltage to Frequency Converter for the Booster Gauss Clock, 25Jul90

[5] H. Weisberg, Acc Div Tech Note No. 145, Gauss Clock Calibration, 13Mar78

\section{Appendix A: Some General Comments, and a Discussion of the Determination of an Experimental Value for $\mathbf{R}_{0}$}

Introduction:

Even after working with these beam and AGS machine variables for 25 years, (ok you now know which of the authors is responsible for this appendix) there is a certain lack of intuition to be overcome regarding their behavior. This appendix describes one aspect of the space, and shows the power of the scan to extract a critical number - the AGS path length or "radius".

The variables are:

1) $\mathrm{F}=$ the $\mathrm{rf}$ frequency (or equivalently the beam revolution frequency) A time interval is required to measure a frequency. The internal machinery of the measuring device will set how wide an interval is required to get a given frequency precision and accuracy.

2) $r=$ the average of the horizontal beam position monitors in the AGS- which for a given monitor means the difference between its reported position and the position which - by survey data and calculation - would result if the beam were on the "Optimal Central Orbit" (OCO) using the terminology of Ed Bleser (Acc Div Tech Note 217, 22jul85). The beam offset from the center of the pick-up electrode can be beam-calibrated, with some assumptions about the dispersion function at the location. The location of the center of the electrode relative to Bleser's OCO is a task for survey (Acc Div Tech Note 452, 'Revisiting the Dead Reckoning of the AGS Orbit Measuring System" Ahrens, 1996). This piece does not claim 'mil' accuracy. The mechanical connection between the PUEs (pick up electrodes the beam sensing plates in the BPMs = beam position monitors) and the magnets is too loose for that. So the value of $\mathrm{R}_{0}$ to be determined here, is not a replacement for Bleser's value in the machine, but is the proper number to use with other numbers reported from the AGS Orbit system (for example the extraction radius for RHIC), that are measured with as few changes or elapsed time from the $\mathrm{R}_{0}$ determination as possible.

3) $\mathrm{GCC}=$ Gauss Clock Counts. One Gauss Clock Count corresponds to a change in the average AGS magnetic field (B) by about .05 Gauss. Reporting a certain number of Gauss Clock Counts requires specifying the rules for defining the associated time interval. Usually only the change in this number as one end of the interval is shifted is important.

The path length the beam follows when it travels on the OCO is expressed as $\left(2 \pi * \mathrm{R}_{0}\right)$. This defines $\mathrm{R}_{0}$. Bleser's analysis based on the magnet properties and survey procedure for the AGS concluded that $\mathrm{R}_{0}=128452.566 \mathrm{~mm}$. 
This appendix deals with the following assumptions and question. Assume the data from the scan (which is the subject of note to which this appendix is attached) have insignificant error bars. Assume that the Gauss Clock system is linear with B i.e. that there are no significant rate or magnitude effects. How is the analysis affected by introducing the wrong ideal path length for the AGS - the wrong $\mathrm{R}_{0}$ ? And given that the analysis is affected, how accurately can we extract a value for $\mathrm{R}_{0}$ from the data within the above assumptions. We can explicitly stay away from measurements taken while the acceleration rate is changing violently. We cannot avoid working with data over a large range in B.

Here we just calculate the set of numbers usually measured. We start with a column of beam momenta, as the beam accelerates from about $2.5 \mathrm{GeV} / \mathrm{c}$ at injection to $30 \mathrm{GeV}$ at extraction. This implies a column of magnetic fields. With the assumption of a linear Gauss Clock, this also implies a column of Gauss Clock Counts. The beam momentum table also implies a column of velocities. Of course because the particles become highly relativistic by the end of acceleration, this velocity table is not at all linear with $\mathrm{P}$. With the assumption of a path length or an $\mathrm{R}_{0}$ (the true value) a column of frequencies results. Needless to say, if we now take this frequency table and turn it back into velocity (using $\mathrm{R}_{0}$ ) and then into a "calculated" momentum, and plot input momentum vs calculated momentum we get a straight line. But let us instead use an incorrect $\mathrm{R}_{0}$ for the calculation of velocity from frequency. Now the connection between input momentum and output momentum is no longer linear. Figure A1 is the result of this exercise for the AGS, with an error introduced into $\mathrm{R}_{0}$ of $+/-1 \mathrm{~mm}$.

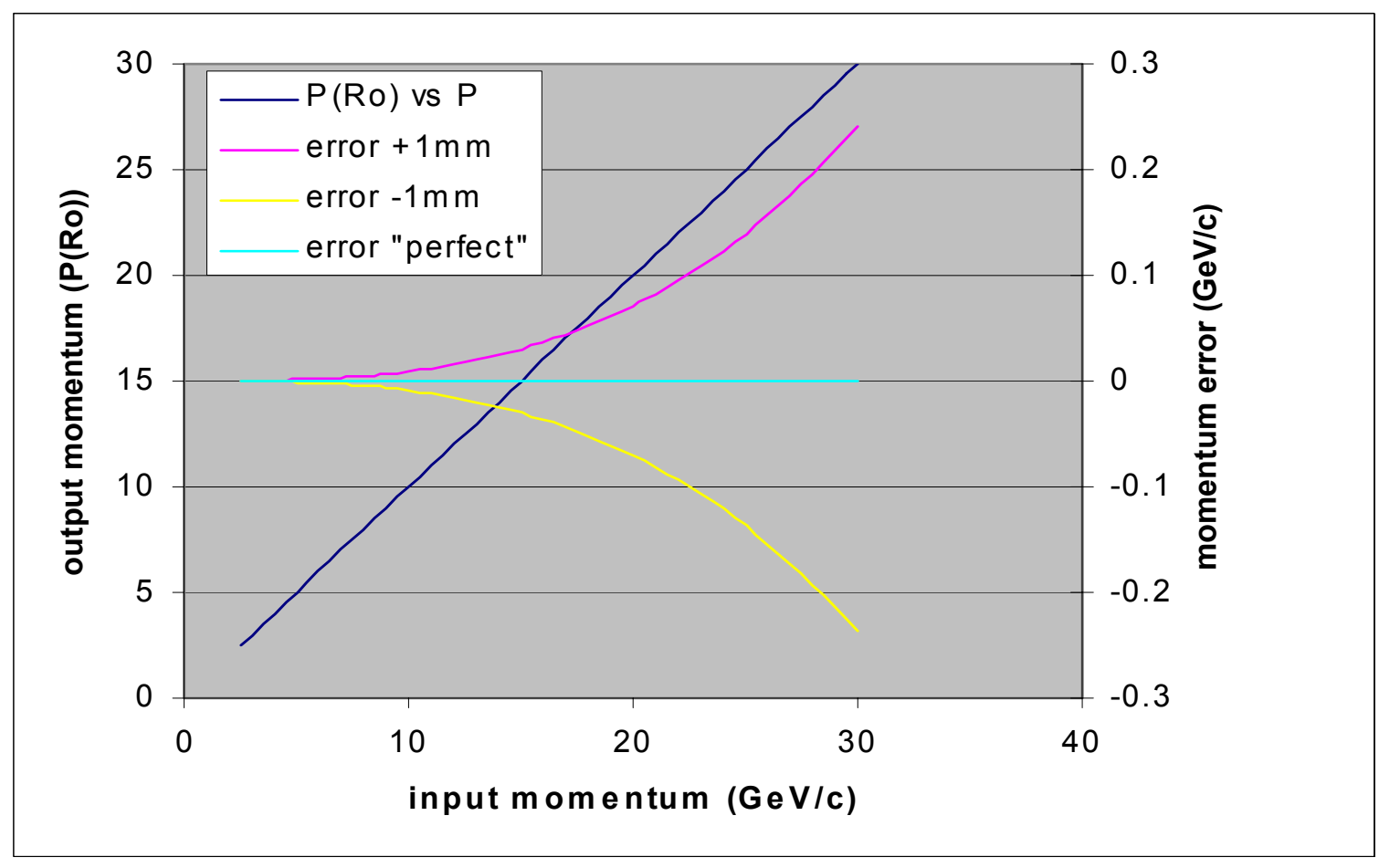

Figure A1 The apparent deviation of calculated beam momentum if the assumed path length is incorrect by $\left(2 \pi^{*}(+/-) 1\right) \mathrm{mm}$.

To connect directly to the units in this note, these momenta can be translated into the AGS magnet field, with some assumed effective radius of curvature. This is given in Figure A2. 


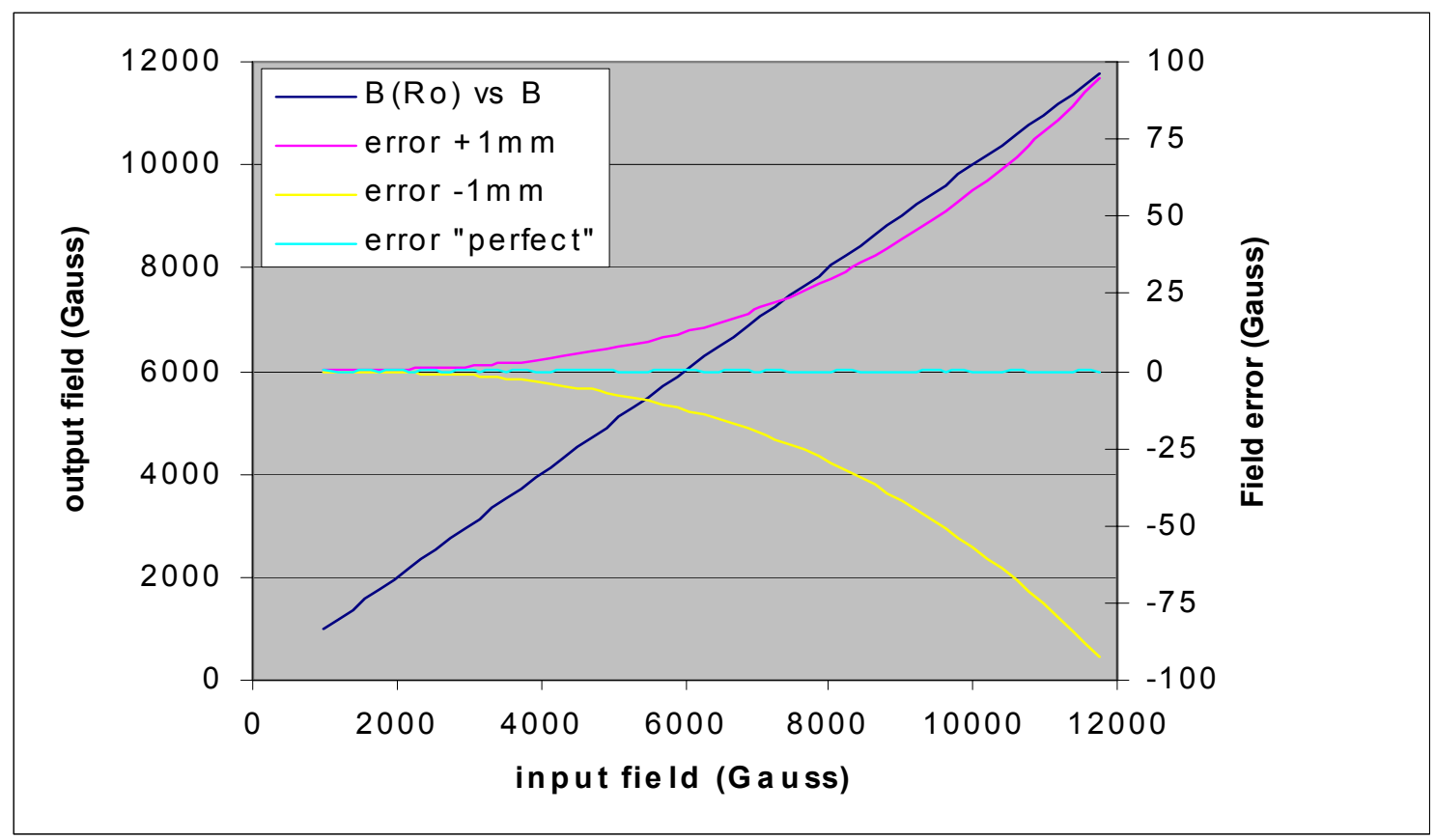

Figure A2 The same simulation data as Figure 1, translated into Magnetic Field

The sensitivity of the result, the deviation from linearity, appears large in this view. The scale (right scale) for the deviation is many tens of Gauss. This is a bit of a cheat however. In the real world, we don't get the true answer, just the wrong one. So it is only the deviation from a straight line that we can use to indicate our error. To make this clear, the "+1mm" simulation data is replotted in Figure A3.

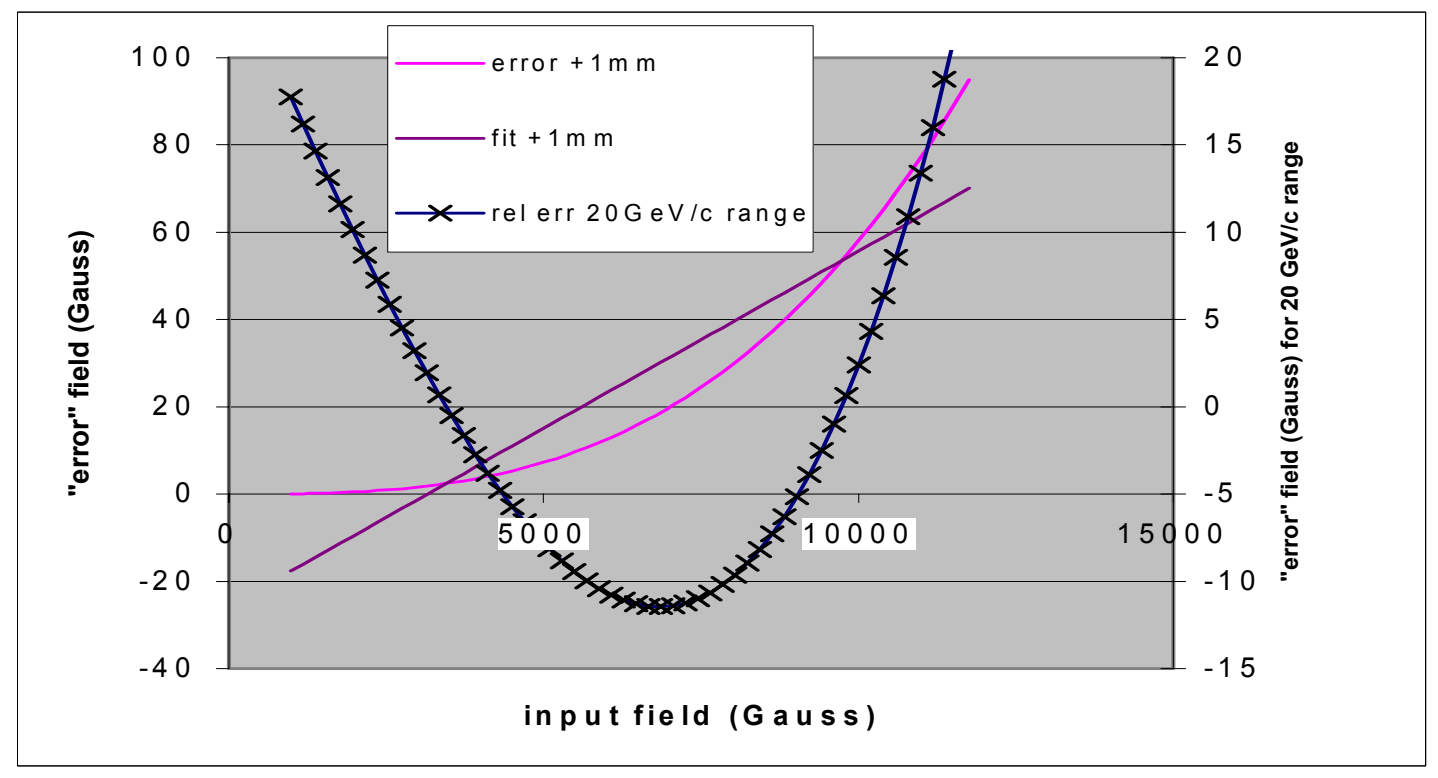

Figure A3 Just looking at the $+1 \mathrm{~mm}$ error assumed in $\mathrm{R}_{0}$ case 
Here the "error" plot is as before. The "fit" indicates how we would process this data - getting a predicted slope of output field against input field that is not zero. The "relative error" curve is the one we know experimentally - the deviation of the resulting processed data from a straight line. Now the relevant variation - the right scale - is reduced to just 10 Gauss. And the situation is even a bit worst for our scan since we only go up to $20 \mathrm{GeV} / \mathrm{c}$. This situation is shown in figure A4.

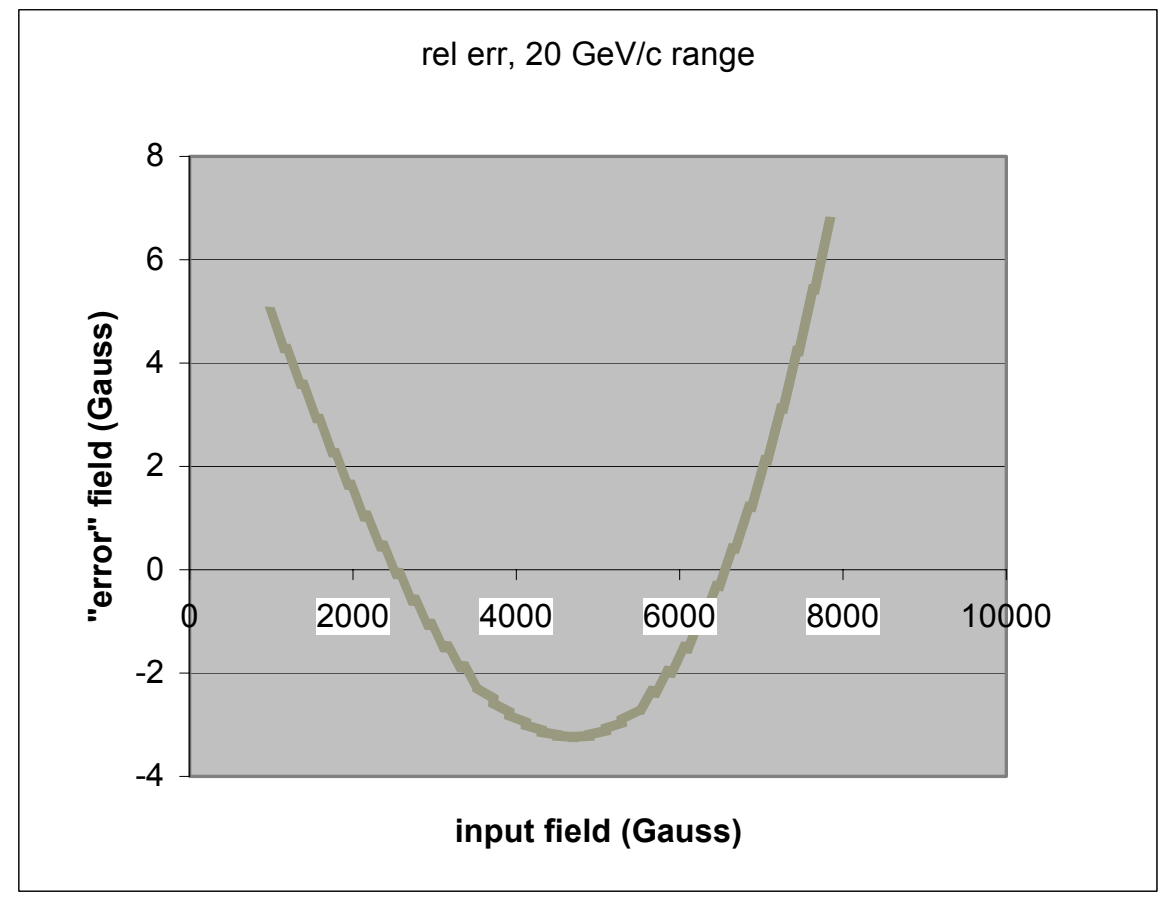

Figure A4 Deviation from Linear Behavior Predicted for a $1 \mathrm{~mm}$ Error in assumed $\mathrm{R}_{0}$

This is the effect, coming from the relativistic nature of the beam as it accelerates, that we can exploit to learn the real $\mathrm{R}_{0}$. To be more careful, we don't really learn anything relevant to "correcting" Bleser's $\mathrm{R}_{0}$. What we learn about is the effective $\mathrm{R}_{0}$ given the "dead reckoned BPM survey numbers, and the set of BPMs contributing to the average. That is the relevant number to use in the calculation for the work of this note. It will change with time. It surely changes a bit over the acceleration cycle. spirit.

Now we can plot the real data from one of the recent runs (the polarized proton run) in the same 


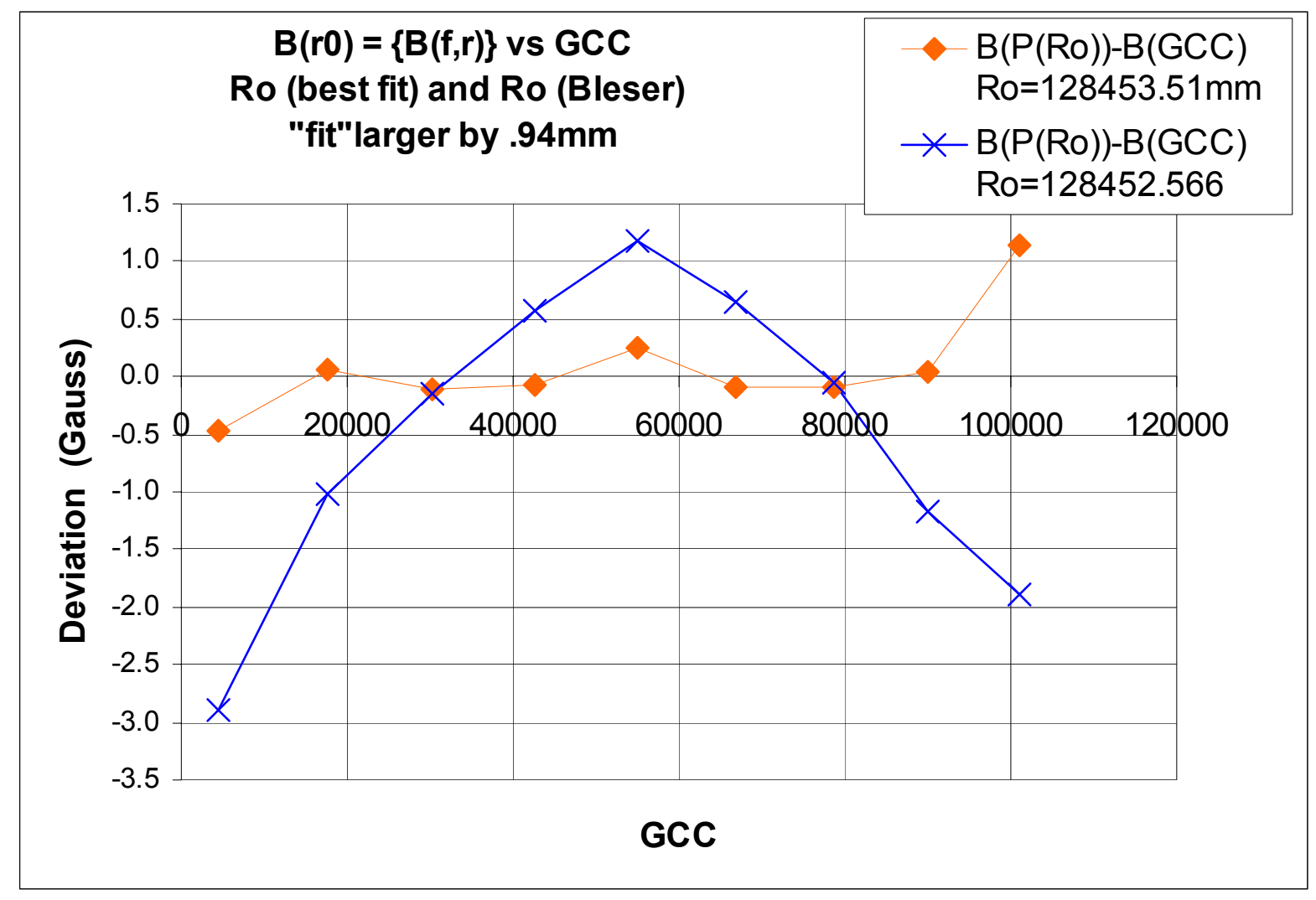

Figure A5 Best Fit for two choices of $\mathrm{R}_{0}$ for the 'polarized proton' scan

This rather subtle change in the assumed $\mathrm{R}_{0}$ then results in a different best fit prediction for the Gauss Clock calibration - which is the point of the exercise for the note in which this appendix is embedded. For the polarized proton scan, the slope changes from $19.50 \mathrm{GCC} / \mathrm{Gauss}$ for the Bleser $\mathrm{R}_{0}$ to 19.455 GCC/Gauss. This $.23 \%$ change is just significant for one use of the calibration, namely putting the extraction porch within .1 unit of 46.5 in $\mathrm{G} \gamma$. 


\section{Appendix B: Exploring the Sensitivities of the Momentum Estimate to (B, R, and F)}

What we are after is an estimate of how well we can extract the beam momentum from our measurements of the beam revolution frequency (really the rf frequency), the magnetic field (really the Gauss clock) and the path length (really the BPM average). The approach we follow is to assume we know one of these variables perfectly, absolutely, and ask how well we determine the momentum given some explicit and hopefully reasonable assumptions for the errors in the other variables.

First, and for some combinations most unintuitive, is the first order response of the machine itself, which is described by the differential relations among these three variables, as the beam accelerates. These are given e.g. in the CERN reference "A Selection of Formulae and Data Useful for the Design of A.G. Synchrotrons" by Bovet et al. CERN/MPS-SI/Int. DL/70/4 1970, page 9. Second, the expected errors on the measurements will sometimes change in a systematic and predictable way during acceleration. Finally, for the requirements of polarized proton acceleration the absolute error in the momentum, rather than the fractional error is the relevant number. An aggressive version of this spec., namely knowledge of momentum to .1 unit of G gamma, is added to the following figures.

Assume we know the radius.

$$
\left.\left.\mathrm{dp} / \mathrm{p}=\gamma^{2} \mathrm{df} / \mathrm{f}\right)_{\mathrm{R}} \text { and } \mathrm{dp} / \mathrm{p}=\mathrm{dB} / \mathrm{B}\right)_{\mathrm{R}}
$$

Predicting the magnetic field is what this note was originally about. $\mathrm{B}=\mathrm{B}_{0}+\mathrm{k}^{*}(\mathrm{GCC})$. For our purposes we assume the error in the prediction is dominated by the error in the assumed slope. $\mathrm{dB} / \mathrm{B}=\mathrm{dk} / \mathrm{k}$, which we take here to be .0025 . In the note we argue the slope is known better than this. (If $\mathrm{k}$ is $1 /(20 \mathrm{GCC} / \mathrm{Gauss})$, a fractional error of .0025 would mean an error in the 20 of .05 . An uncertainty in the injection field $\mathrm{B}_{0}$ and the least count in the GCC also contribute some error, which we ignore here.

The frequency error comes from two effects. The meter necessarily measures over a time interval. The error in the measured frequency scales as the inverse of this window. The meter reports this inherent uncertainty. The frequency being measured also varies systematically over this time interval and, for a uniform acceleration situation, that variation is not strictly linear. The average frequency over the window, which is what the frequency meter reports and we use, underestimates the "instantaneous" frequency just in the middle of the time interval. As the particles become more relativistic, the deviation from linearity gets smaller; and a longer window is justified. For the latter part of the cycle the frequency meter reported error is about $2 \mathrm{~Hz}$ out of $2 \mathrm{MHz}$. This is a standard deviation I suspect. In the analysis I take $4 \mathrm{~Hz}$ as the error near the end, and pretend we increase the width at earlier times according to a procedure to keep the actual variation across the interval constant. So the measuring width gets decreased near injection at the expense of reported error in this model. The frequency error estimates are somewhat overestimates of the errors occurring during the scan. The upshot of this analysis is given in figure B1. The sign of the error is not important. Appendix C revisits this subject. The error could be reduced until shot-to-shot variations dominated the answer. The study of shot-to-shot variations, and the extent to which these can be dealt with is future work. 


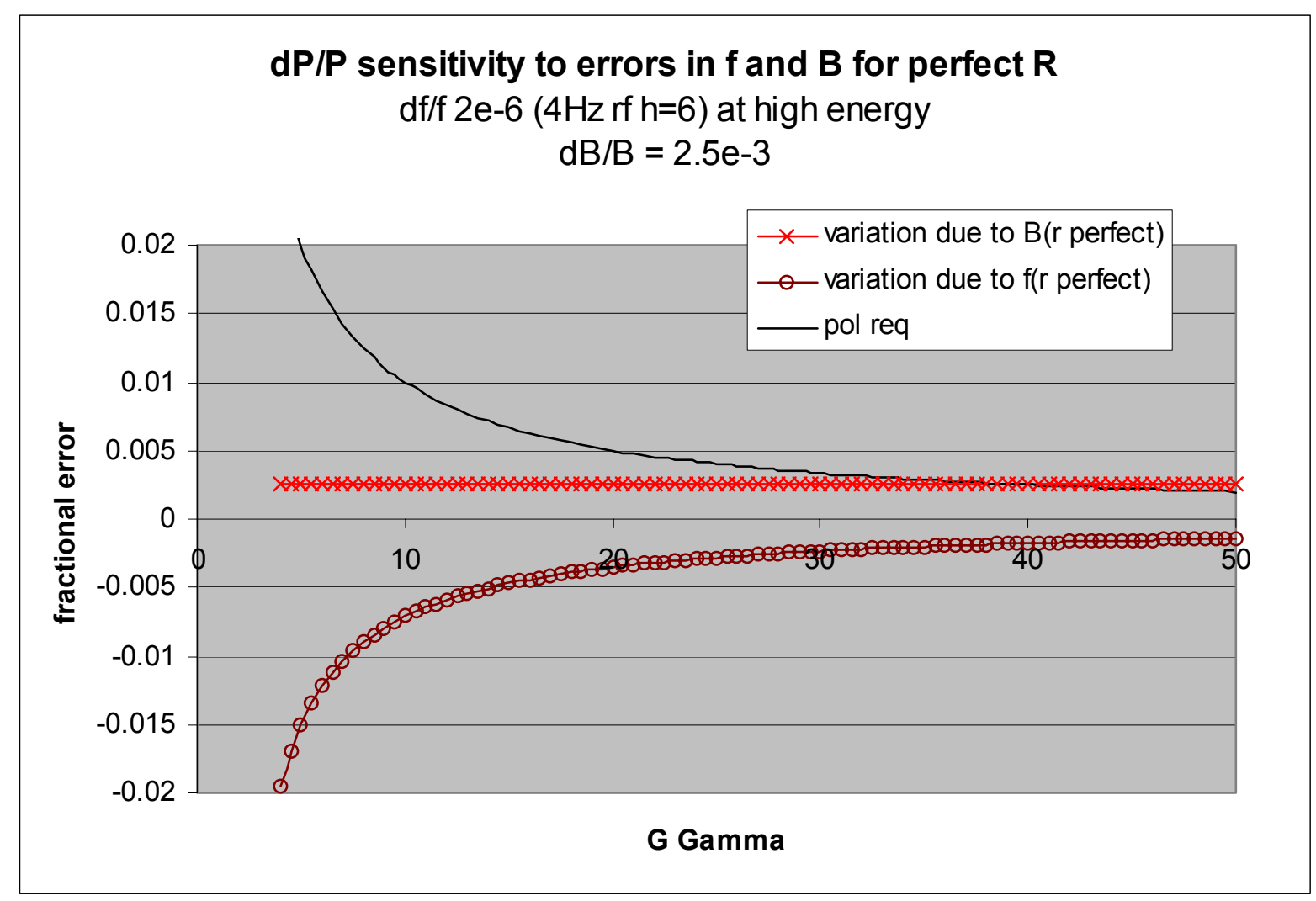

Figure B1 Sensitivity of Momentum in the AGS to Uncertainty in $\mathrm{f}$ or B at a Known R

So this would claim, if our estimate of errors is right, that $(R, f)$ beats $(R, B)$ for energy above transition. In fact as mentioned above, the frequency error is not maxed out.

Off we go.

Assume we know the field.

$$
\left.\left.\mathrm{dp} / \mathrm{p}=\left\{\left(\gamma^{2} \gamma_{\mathrm{tr}}^{2}\right) /\left(\gamma_{\mathrm{tr}}^{2}-\gamma^{2}\right)\right\} \mathrm{df} / \mathrm{f}\right)_{\mathrm{B}} \text { and } \mathrm{dp} / \mathrm{p}=\gamma_{\mathrm{tr}}^{2} \mathrm{dR} / \mathrm{R}\right)_{\mathrm{B}}
$$

We have not estimated the radial error yet. For now we take this as $1 \mathrm{~mm}$. From appendix A analysis this doesn't seem too large, but it would be hard to prove we do say 5 times better. Figure B2 gives the predicted sensitivities. 


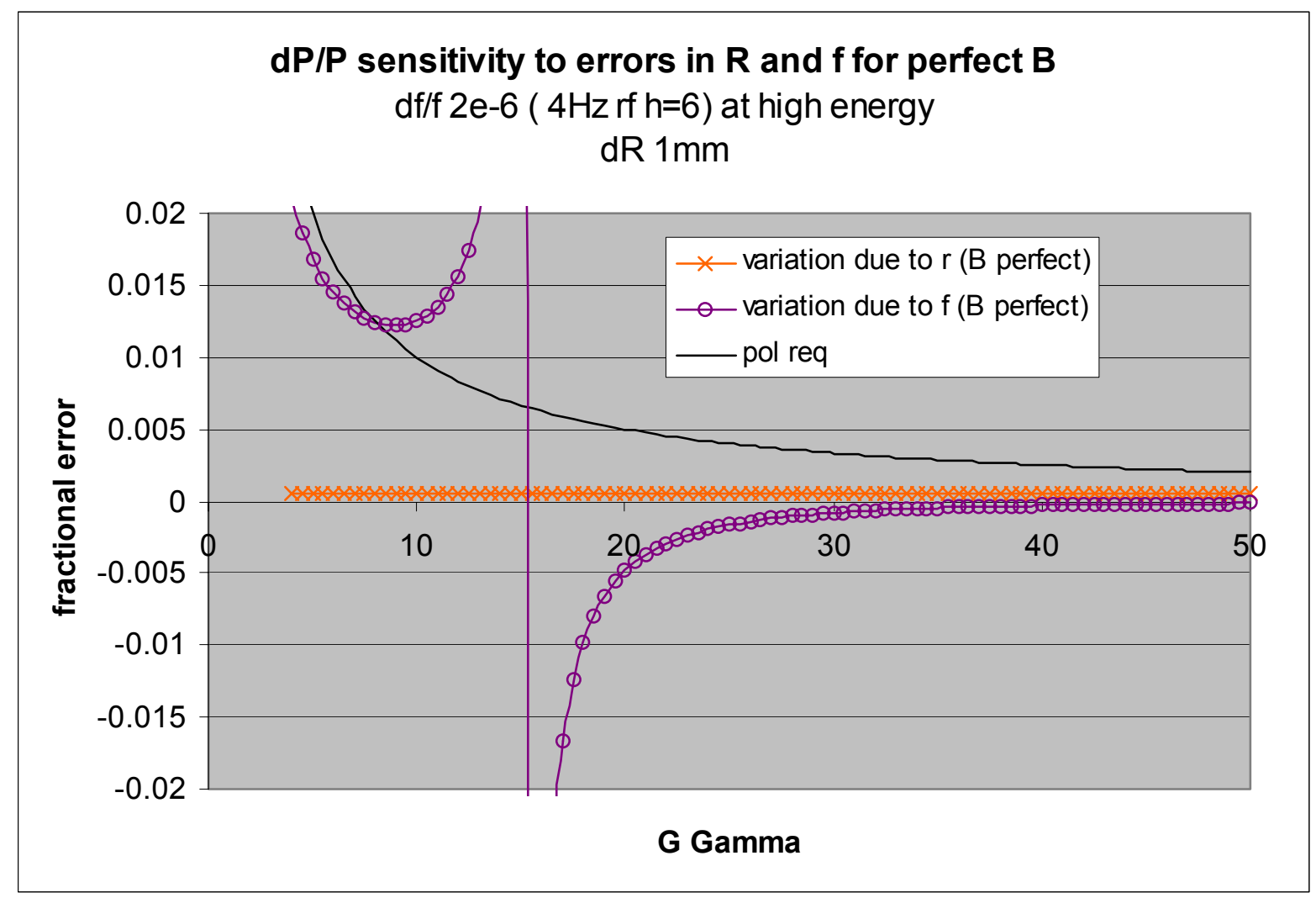

Figure B2 Sensitivity of Momentum to Uncertainty in $\mathrm{f}$ and $\mathrm{r}$ at known B

Clearly f doesn't help much until well after transition if one only knows B, though it does very well at high field. Knowing B (perfectly) above transition is enough to know p even with errors in the other variables that are four times our estimates.

Finally, assume we know the frequency.

$$
\left.\left.\mathrm{dp} / \mathrm{p}=\left\{\left(-\gamma^{2}\right) /\left({\gamma_{\mathrm{tr}}}^{2}-\gamma^{2}\right)\right\} \mathrm{dB} / \mathrm{B}\right)_{\mathrm{f}} \text { and } \mathrm{dp} / \mathrm{p}=\gamma^{2} \mathrm{dR} / \mathrm{R}\right)_{\mathrm{f}}
$$

Assuming the same errors as above, the resulting variation with energy is given in figure B3. 


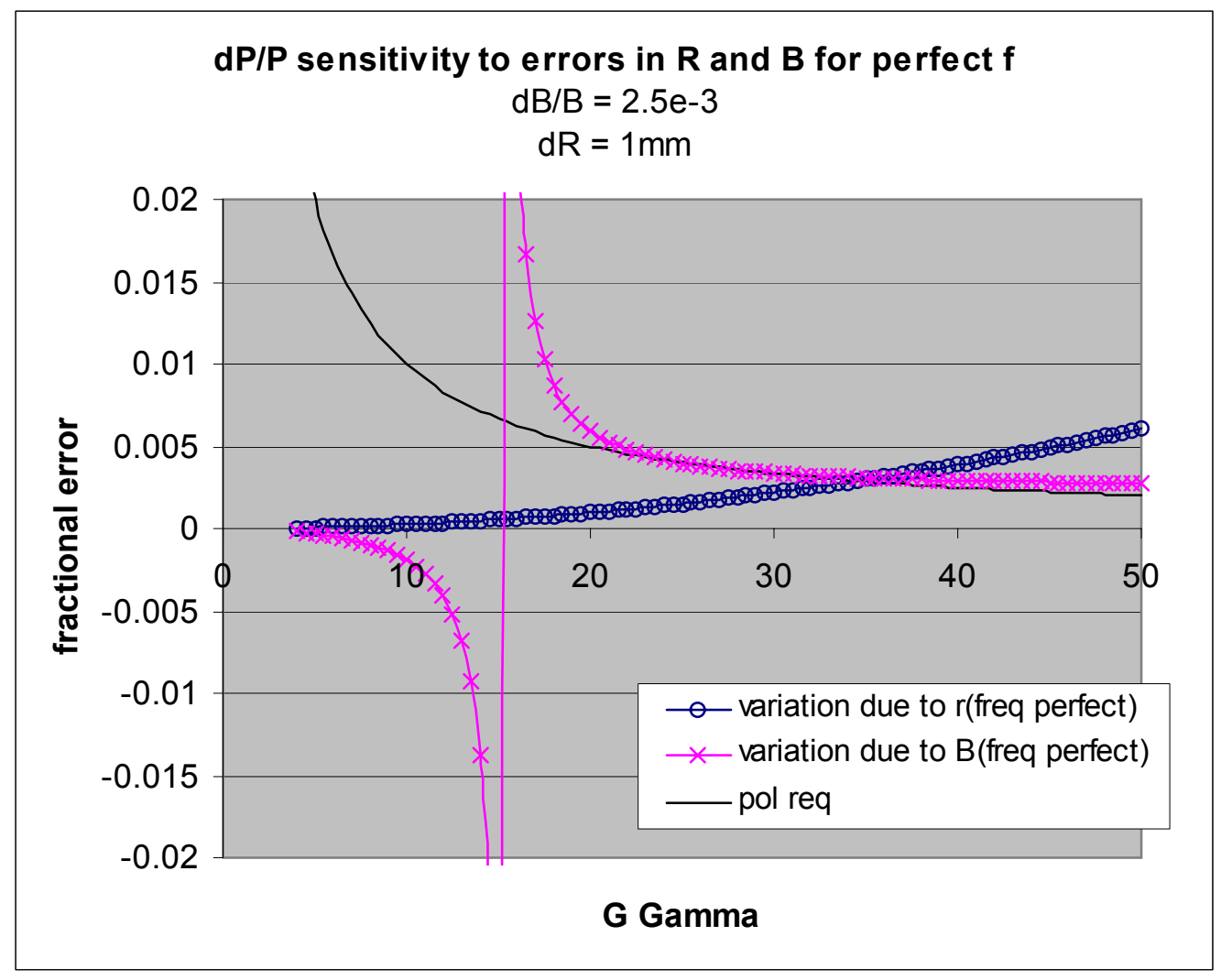

Figure B3 Sensitivity of Momentum to Uncertainty in R and B at known Frequency

This one starts from the only variable that comes essentially precalibrated (ok assuming the frequency meter's internal clock is happy). Again the beam momentum is not well defined at transition from $f$ and $B$, but at low energy this is ok. And at low energy the variation due to radial uncertainty is also excellent. At injection, a very careful frequency measurement can essentially stand alone. The radius cannot be off enough to cause a problem if the beam is within the machine aperture. 


\section{Appendix C Fine Tuning the Frequency Measurement}

As this note has evolved, it becomes important to dot some i's just because of the accuracy being demanded of some of the extracted numbers. Here we attempt to shore up the uncertainty in the measured frequencies. The frequency measured is the frequency being sent to the accelerating cavities. It is ' $h$ ' times the beam revolution frequency, where $h$ was 12 during the polarized run in Dec 01 , and 6 during the subsequent high intensity run.

Inherent in a frequency measurement is a time interval across which the measurement is made. The longer this interval, the more accurate the measure of the average frequency. The frequency meter indicates the order of this uncertainty just by the number of significant digits it displays. A completely different error source results from the fact that the AGS will not be exactly the same from cycle to cycle. This error can be estimated by comparing several measurements, which would ideally be identical. Again the frequency meter computes the standard deviation over the measurements. Typically 10 cycles are averaged over.

The other aspect of the frequency measurement is associated with the fact that the beam is (usually) accelerating during the measurement and so the frequency is changing. This gives a systematic shift between the reported average frequency and the frequency at the center of the interval. The procedure is to take measurements over a time interval ending at the desired time, and then over an equal interval starting at the desired time. The frequency reported is the average of these two measurements. For measurements reported here the time interval was always a fixed $1 \mathrm{~ms}$ for each interval. (This is equivalent to using a $2 \mathrm{~ms}$ interval centered on the measurement time). The question is what is the associated systematic offset in the reported frequency from the true value at the center. If the frequency were constant, or increasing linearly with time, the answer would be zero - no error. In fact the rate of change of frequency is decreasing, as the particles become more relativistic. It is this change that is being quantified here.

To an excellent approximation, over the windows of interest, the acceleration rate is constant. That is, $\mathrm{dB} / \mathrm{dt}$ or $\mathrm{dP} / \mathrm{dt}$ or $\mathrm{d}(\beta \gamma \mathrm{m}) / \mathrm{dt}$ is a slowly varying function for 100's of milliseconds once acceleration gets going until the slow down onto the porch (see figure 2 in the main document showing Bdot over the cycle. The frequency varies linearly with the beam velocity, or with $\beta$, so understanding $\beta$ 's time dependence - deviation from linear increase - is sufficient. Translation from $\beta$ to frequency means multiplication by $\left(\mathrm{h}^{*} \mathrm{c} / 2 \pi \mathrm{R}\right)=\mathrm{F}^{\mathrm{rf}}$, (which is a little above $4 \mathrm{MHz}$ for $\mathrm{h}=12$ ) the rf frequency a particle traveling at the speed of light would have.

We expand $\beta$ in a Taylor's series around our measuring point, keeping the quadratic in $\mathrm{t}$ term, for a situation where $d P / d t$ is constant $\left(=k^{*} t\right)$. This is only tedious since $P=m^{*}\left(\beta / \sqrt{ }\left(1-\beta^{2}\right)\right)$, is completely defined in terms of $\beta$ and the particle rest mass $m$.

We get the answer:

$$
\beta=\beta(0)+\left\{\left(\mathrm{m}^{2} / \mathrm{E}^{3}\right) * \mathrm{k}\right\} \mathrm{t}+\left\{\left(-(3 / 2) \mathrm{m}^{2} \mathrm{P} / \mathrm{E}^{5}\right) * \mathrm{k}^{2}\right\} \mathrm{t}^{2}
$$

where $\mathrm{E}$ and $\mathrm{P}$ are evaluated at the point of interest, at $\mathrm{t}=0$. 
The integral of $\beta$ over an interval from 0 to $\mathrm{T}$ is then

${ }_{0} \int^{\mathrm{T}}(\beta \mathrm{dt})=\beta(0) * \mathrm{~T}+\left\{\left(\mathrm{m}^{2} / \mathrm{E}^{3}\right) * \mathrm{k}\right\}\left(\mathrm{T}^{2} / 2\right)+\left\{\left(-(3 / 2) \mathrm{m}^{2} \mathrm{P} / \mathrm{E}^{5}\right) * \mathrm{k}^{2}\right\}\left(\mathrm{T}^{3} / 3\right)$

The frequency meter does this integral for an interval above $\mathrm{t}=0$ and one below $\mathrm{t}=0$. The second term, going as $\mathrm{T}^{2}$, cancels if an average of the two interval evaluations is taken. The third terms from the two intervals add and reduce the reported frequency relative to the value at $t=0$. The frequency difference between the two intervals is then given by:

$$
\Delta=\mathrm{F}^{\mathrm{rf}}{ }_{\mathrm{c}}(1 / \mathrm{T})\left\{\left(\mathrm{m}^{2} / \mathrm{E}^{3}\right) * \mathrm{k}\right\} \mathrm{T}^{2}=\mathrm{F}^{\mathrm{rf}}{ }_{\mathrm{c}}\left\{\left(\mathrm{m}^{2} / \mathrm{E}^{3}\right) * \mathrm{k}\right\} * \mathrm{~T}
$$

and the required correction to the reported frequency is:

$$
\mathrm{f}_{\text {corr }}=\mathrm{F}^{\mathrm{rf}}{ }_{\mathrm{c}}(2 / \mathrm{T})\left\{\left((3 / 2) \mathrm{m}^{2} \mathrm{P} / \mathrm{E}^{5}\right)^{*} \mathrm{k}^{2}\right\}\left(\mathrm{T}^{3} / 3\right)=\left(\mathrm{EP} / \mathrm{m}^{2}\right)^{*}\left\{\Delta / \mathrm{F}^{\mathrm{rf}}{ }_{\mathrm{c}}\right\}^{*} \Delta
$$

Next we put in some general numbers for our situation. Injection into the AGS for polarized protons is at kinetic energy of $1.5 \mathrm{GeV}$. Extraction is at about $24 \mathrm{GeV}$. The momentum slope with Westinghouse is roughly $25 \mathrm{GeV} / \mathrm{c} / \mathrm{sec}$. Take $\mathrm{F}^{\mathrm{rf}}{ }_{\mathrm{c}}$ to be $4 \mathrm{MHz}$. and the integration window to be $1 \mathrm{~ms}$. Then $\Delta$ varies from $6 \mathrm{kHz}$ to $6 \mathrm{~Hz}$.

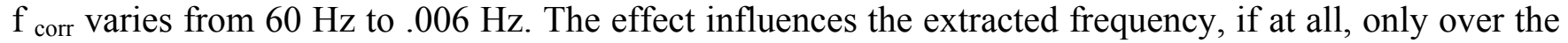
lower portion of the cycle. For the first bin of the polarized measurement, the measured $\Delta$ is $5220 \mathrm{~Hz}$ and the reported $\mathrm{rf}$ frequency is $4,231,850+/-200 \mathrm{~Hz}$; $\mathrm{k}=36.6 \mathrm{GeV} / \mathrm{c} / \mathrm{sec}$. This analysis specifically for this measurement gives a predicted $\Delta$ of $5350 \mathrm{~Hz}$ and $\mathrm{f}_{\text {corr }}$ of $62.8 \mathrm{~Hz}$. That we get the $\Delta$ right is a necessary but not sufficient check that the correction term is right. Factors of two come and go easy. Nevertheless, applying this correction to the data results in an increase in the predicted field for the lowest (most sensitive) point of about .15 Gauss. This is marginally below the resolution relevant for the analysis of this paper. 